\title{
LEAVABLE GAMBLING PROBLEMS WITH UNBOUNDED UTILITIES
}

\author{
A. MAITRA, R. PURVES, AND W. SUDDERTH
}

\begin{abstract}
The optimal return function $U$ of a Borel measurable gambling problem with a positive utility function is known to be universally measurable. With a negative utility function, however, $U$ may not be so measurable. As shown here, the measurability of $U$ for all Borel gambling problems with negative utility functions is equivalent to the measurability of all PCA sets, a property of such sets known to be independent of the usual axioms of set theory. If the utility function is further required to satisfy certain uniform integrability conditions, or if the gambling problem corresponds to an optimal stopping problem, the optimal return function is measurable. Another return function $W$ is introduced as an alternative to $U$. It is shown that $W$ is always measurable and coincides with $U$ when the utility function is positive.
\end{abstract}

\section{INTRODUCTION}

The definition of a Borel gambling problem will be given in this section along with the statements of our major results. Most of our notation and definitions are adapted from Dubins and Savage [11].

The term Borel set will be used here to mean a Borel subset $X$ of a Polish space. Denote by $\underset{\sim}{B}(X)$ the Borel subsets of any Borel set $X$ and by $\mathscr{P}(X)$ the set of countably additive probability measures defined on $X$. If $\mathscr{P}(X)$ is given the usual weak topology, then it too has the structure of a Borel set and its Borel sigma-field coincides with the smallest sigma-field such that $\gamma \rightarrow \gamma(A)$ is a measurable function from $\mathscr{P}(X)$ to the real line $R$ for each $A$ in $\underset{\sim}{B}(X)$. (See, for example, Chapter II of Parthasarathy [20], Chapter III (60 to 62) of Dellacherie and Meyer [9], and Dubins and Freedman [10] for information about the weak topology and the Borel structure of $\mathscr{P}(X)$.) A function $f: X \rightarrow$ $Y$ from one Borel set to another is called universally measurable if $f^{-1} \underset{\sim}{(\underset{\sim}{X}(Y))}$ is contained in the completion of $\underset{\sim}{B}(X)$ under every measure in $\mathscr{P}(X)$.

Let $F$ be a nonempty Borel set to be regarded as the set of possible fortunes of a gambler or possible states of a system. A Borel gambling house is a mapping

Received by the editors October 3, 1988.

1980 Mathematics Subject Classification (1985 Revision). Primary 60G40, 62L15, 93E20, 03 E35.

Key words and phrases. Measurable gambling, réduite, optimal stopping, measurable strategies, analytic sets, PCA sets.

Research by W. S. supported by National Science Foundation Grant DMS-8801085. 
$\Gamma$ from $F$ to the collection of nonempty subsets of $\mathscr{P}(F)$ such that the set $\Gamma=\{(x, \gamma): \gamma \in \Gamma(x)\}$ is a Borel subset of the product $F \times \mathscr{P}(F)$. An element $\gamma$ of $\Gamma(x)$ is thought of as a gamble at $x$ and represents a possible distribution for the next state of a player whose current state is $x$.

A strategy $\sigma$ is a sequence $\sigma_{0}, \sigma_{1}, \ldots$, where $\sigma_{0} \in \mathscr{P}(F)$ and, for $n>0, \sigma_{n}$ is a universally measurable map from $F^{n}$ into $\mathscr{P}(F)$. Let $H$ be the countably infinite product $F \times F \times \cdots$, and use $h=\left(h_{1}, h_{2}, \ldots\right)$ to denote a typical element or history in $H$. A strategy $\sigma$ determines a measure $\mu(\sigma)$ on $\underset{\sim}{B(H)}$. Namely, the $\mu(\sigma)$-marginal distribution on the first coordinate is $\sigma_{0}$, and, given $\left(h_{1}, \ldots, h_{n}\right)$, the $\mu(\sigma)$-conditional distribution of $h_{n+1}$ is $\sigma_{n}\left(h_{1}, \ldots, h_{n}\right)$. We will usually write $\sigma$ rather than $\mu(\sigma)$ for this measure.

A strategy $\sigma$ is available at $x$ in $\Gamma$ if $\sigma_{0} \in \Gamma(x)$ and, for every $\left(h_{1}, \ldots, h_{n}\right)$, $\sigma_{n}\left(h_{1}, \ldots, h_{n}\right) \in \Gamma\left(h_{n}\right)$. Intuitively, a gambler must choose, at every stage of play, a gamble at the current state and this gamble corresponds to the distribution of the next state. Let $\Sigma(x)$ be the collection of all strategies available at $x$. By identifying each $\sigma$ with the measure $\mu(\sigma)$, we can regard $\Sigma(x)$ as a subset of $\mathscr{P}(H)$.

A stop rule $t$ is a Borel mapping from $H$ to $N=\{1,2, \ldots\}$ such that $[t=n]$ belongs to the sigma-field generated by the first $n$ coordinate functions on $H$ for every $n$. (Notice that we require $t(h)<\infty$ for every $h \in H$.) A policy $\pi$ at $x$ is a pair $(\sigma, t)$ where $\sigma \in \Sigma(x)$ and $t$ is a stop rule.

Let $u$ be a Borel function from $F$ to $R$ to be regarded as the utility function. The utility of a policy $\pi$ is defined to be the expected utility under $\sigma$ at the time of stopping; that is,

$$
u(\pi)=\int u\left(h_{t}\right) d \sigma
$$

We will assume that $u(\pi)$ is well defined as an integral for every $\pi$ available at every $x$. This is, of course, the case if $u$ is bounded above or below.

The triple $(F, \Gamma, u)$ is a Borel gambling problem and its optimal return function is defined, for $x \in F$, as

$$
U(x)=\text { the maximum of } \sup u(\pi) \text { and } u(x),
$$

where the supremum is over policies $\pi$ at $x$. We will always assume in the sequel that $\Gamma$ is leavable in the sense that the point mass $\delta(x)$ is in $\Gamma(x)$ for every $x \in F$. There is no loss of generality for the purposes of this paper because $U$ remains the same if $\delta(x)$ is adjoined to $\Gamma(x)$ for every $x$. It will also be assumed for ease of exposition that $U$ is everywhere finite and, in particular, that $u(\pi)<\infty$ for every available policy $\pi$. This assumption entails no real loss of generality but makes certain proofs less tedious.

Once we have assumed $\Gamma$ to be leavable, we can also restrict attention to Borel measurable strategies $\sigma$. This is because, for every $x$ and $\sigma \in \Sigma(x)$, there is a strategy $\sigma^{\prime} \in \Sigma(x)$ such that $\sigma_{0}^{\prime}=\sigma_{0}$ and, for $n \geq 1, \sigma_{n}^{\prime}$ is a Borel measurable function equal to $\sigma_{n}$ on a Borel set of $\sigma$-measure one and such 
that $\sigma_{n}^{\prime}\left(h_{1}, \ldots, h_{n}\right)=\delta\left(h_{n}\right)$ elsewhere. Clearly, $\sigma$ and $\sigma^{\prime}$ determine the same measure $\mu(\sigma)$ on $B(H)$.

By the way, there would be no real gain in generality and no change in $U$ if we permitted universally measurable stop rules. This is because, given $\sigma$ and such a stop rule $t$, it is not difficult to find a Borel stop rule $t^{\prime}$ such that $\sigma\left[t=t^{\prime}\right]=1$.

In the original formulation of gambling theory given by Dubins and Savage [11], gambles were taken to be finitely additive measures defined on all subsets of $F$ and strategies were not restricted to be measurable. In this general, nonmeasurable setting, a fundamental result is that the optimal return function is the least function $Q: F \rightarrow R$ such that

$$
\begin{gathered}
Q \geq u ; \\
\int Q d \gamma \leq Q(x) \quad \text { for all } x \in F \text { and } \gamma \in \Gamma(x) .
\end{gathered}
$$

A function $Q$ which satisfies (ii) is called excessive.

Dubins and Savage assumed the utility function $u$ to be bounded. However, many of their results, including the characterization of the optimal return function just stated, remain true when $u$ is nonnegative or nonpositive. For a Borel problem, if there is a least universally measurable $Q$ satisfying (i) and (ii), it is called the réduite or reduced function of $u$.

Dubins and Savage raised the question of whether the optimal return function for a measurable problem is measurable and whether a gambler can do as well with measurable strategies as with nonmeasurable ones. It is equivalent to ask whether the function $U$, defined above as the optimal return from measurable strategies, is measurable and is the reduced function of $u$. These questions were answered positively by Strauch [22] for Borel problems with a bounded utility function $u$ and later by Dubins and Sudderth [13] and Dellacherie and Meyer [9] for $u \geq 0$ :

Theorem 1.1. For a Borel problem with a nonnegative $u, U$ is universally measurable and is the reduced function of $u$.

In fact, the conclusion of Theorem 1.1 will be shown to hold not only for nonnegative $u$ but also as long as the process $\left\{u^{-}\left(h_{n}\right)\right\}$ is uniformly integrable under each $\sigma$ available. (Here $u^{-}=(-u) \vee 0$.)

The major result of this paper is that the situation is quite different for general nonpositive $u$. (Notice that a nonpositive $u$ corresponds to a nonnegative loss function and is a common assumption in decision problems.) For the statement of the theorem, recall that an analytic set is the continuous image of a Borel set and a PCA set is the continuous image of the complement of an analytic set.

Theorem 1.2. For a general Borel problem with a nonpositive $u$, the statement " $U$ is universally measurable" is independent of the axioms of Zermelo-Fraenkel 
set theory together with the axiom of choice (ZFC). More specifically:

(i) If all PCA sets are universally measurable, then $U$ is universally measurable and is the reduced function of $u$.

(ii) If not all PCA sets are universally measurable, then $U$ need not be universally measurable and there need not exist a reduced function of $u$.

Martin and Solovay [17] showed that the statement "all PCA sets are measurable" is provable in the theory ZFC + Martin's axiom $+2^{\aleph_{0}}>\aleph_{1}$, which is known to be consistent if $\mathrm{ZF}$ is. On the other hand, the negation of the statement is provable in ZFC + axiom of constructibility (ZFL) (cf. Chapter 5 of Moschovakis [19]), which too is consistent if ZF is, as was shown by Gödel.

An interesting class of gambling problems is the stop-or-go problems in which, at each $x \in F$, a gambler has available at most one gamble $\gamma(x)$ other than the trivial, point mass $\delta(x)$ at $x$. These problems correspond to optimal stopping problems [12]. Even for these special problems, there is a difference in behavior for $u \geq 0$ and $u \leq 0$.

Theorem 1.3. For a Borel stop-or-go problem, $U$ is universally measurable and is the reduced function of $u$. If $u \geq 0, U$ is Borel measurable. For $u \leq 0$, it can happen that $U$ is not Borel.

Here is how the rest of the paper is organized: $\S 2$ has two lemmas about conditional distributions. $\S 3$ introduces the class of upper analytic functions and collects some of their properties which we will need. In $\S 4$ we study a hierarchy of return functions $U_{\alpha}$, where $\alpha$ is an ordinal number. It is shown that $U_{\alpha}$ is universally measurable for countable ordinals $\alpha$ and that, if $u \geq 0$, then $U=$ $U_{\omega}$, where $\omega$ is the first infinite ordinal (Theorem 4.8). In general, $U=U_{\omega_{1}}$, where $\omega_{1}$ is the first uncountable ordinal. Our study of $U_{\omega_{1}}$ depends critically on a result of Moschovakis from effective descriptive set theory, and this result is presented in $\S 5$. After $\S 5$ we are ready to prove the positive assertions of Theorems 1.2 and 1.3, and these proofs are in $\S \S 6$ and 7, respectively. The negative assertions are based on an example and modifications of it which are presented in $\S 8$. The example of $\S 8$ does not depend on the intervening sections and can be read immediately. An alternative return function $W$ is defined in $\S 9$ and shown to be universally measurable.

\section{TWO LEMMAS ON BOREL SETS AND CONDITIONAL DISTRIBUTIONS}

Let $X$ and $Y$ be Borel sets and, for $\mu \in \mathscr{P}(X \times Y)$, let $\mu_{0}$ denote the marginal distribution of $\mu$ on $X$ and let $\mu_{1}$ be a version of the regular conditional $\mu$-distribution of $y$ given $x$. Notice that $\mu_{1}$ is a measurable map from $X$ to $\mathscr{P}(Y)$.

Lemma 2.1. For each Borel subset $E$ of $\mathscr{P}(Y)$ the mapping $\psi: \mathscr{P}(X \times Y) \rightarrow$ $[0,1]$, defined by

is Borel measurable.

$$
\psi(\mu)=\mu_{0}\left\{x: \mu_{1}(x) \in E\right\},
$$


Proof. See Lemma 2.2 of Sudderth [23].

In the sequel, we will be working with families of probability measures on a measurable space and will require conditional distributions which are jointly measurable functions of the underlying measure in the family and the conditioning variable. Such conditional distributions exist by the next lemma.

Lemma 2.2. There is a Borel measurable mapping $\nu: \mathscr{P}(X \times Y) \times X \rightarrow \mathscr{P}(Y)$ such that, for every $\mu \in \mathscr{P}(X \times Y), \nu(\mu, x)$ is a regular conditional $\mu$-distribution for $y$ given $x$.

Proof. This is clear if $Y$ is countable. If $Y$ is uncountable, the argument is simply a matter of retracing the steps in the construction of a regular conditional probability and checking measurability at each step. To begin with, take $Y$ to be $2^{N}$, the space of all infinite sequences of 0 's and 1's. The general case can be obtained from this one by using a Borel isomorphism. Let $F$ be the algebra of subsets of $2^{N}$ which depend on finitely many coordinates. For the next step, let $B_{1}, B_{2}, \ldots$ be a sequence of Borel sets in $X$ which generates the Borel sigma-algebra there, and for each $n$, let $\underset{\sim}{B_{n}}$ be the algebra generated by $B_{1}$, $\ldots, B_{n}$.

Let $\mu \in \mathscr{P}(X \times Y)$ and $F \in F$. It is straightforward to write down an explicit expression in $\mu, x, F$ which gives the $\mu$-probability of the set $X \times F$, given the algebra $\underset{\sim}{B}$, evaluated at $x \in X$. (If $x$ belongs to an atom $A$ of $\underset{\sim}{B}$ for which $\mu(A \times Y)=0$, take the conditional probability, for example, to be $\mu(X \times F)$.) Using $\rho_{n}(\mu, x, F)$ as an abbreviation for the expression, it is easy to check that, for each $F \in \underset{\sim}{F}$, the function $(\mu, x) \rightarrow \rho_{n}(\mu, x, F)$ is jointly measurable in $\mu$ and $x$, and for each $\mu, x$ the function $F \rightarrow \rho_{n}(\mu, x, F)$ is a countably additive probability on $F$.

Now define $\rho(\mu, x, F)$ to be the limit of the numbers $\rho_{n}(\mu, x, F), n=$ $1,2, \ldots$, on the set of $(\mu, x)$ where $\limsup \rho_{n}(\mu, x, G)=\liminf \rho_{n}(\mu, x, G)$ for every $G \in \underset{\sim}{F}$; and define it to be $\mu(X \times F)$ otherwise. Again $\rho(\mu, x, F)$ is jointly measurable in $\mu$ and $x$. Also, for each $\mu, x, \rho(\mu, x, \bullet)$ is a finitely additive probability on $\underset{\sim}{F}$. Then the special properties of $\underset{\sim}{F}$ ensure that $\rho(\mu, x, \bullet)$ is countably additive on $\underset{\sim}{F}$. Finally, set $\nu(\mu, x)$ to be the unique countably additive extension of $\rho(\tilde{\mu}, x, \bullet)$ to the Borel sets in $Y$.

Then $\nu$ is measurable from $\mathscr{P}(X \times Y) \times X$ to $\mathscr{P}(Y)$, and so for each $\mu$, the section $\nu_{\mu}: x \rightarrow \nu(\mu, x)$ is measurable, which establishes the measurability clause in the definition of a conditional distribution. A martingale convergence theorem can be used to verify the other clause.

\section{SOME PROPERTIES OF UPPER ANALYTIC FUNCTIONS}

Let $f$ be a function from the Borel set $X$ into the extended-real-line $\bar{R}$. Say that $f$ is upper analytic if, for every $c \in R$, the set $\{x: f(x)>c\}$ is analytic. Upper analytic functions are universally measurable because analytic 
sets are universally measurable and Borel functions are upper analytic because Borel sets are analytic. (Kuratowski [14]). These functions arise quite naturally in the theory of measurable gambling because the optimal return function $U$ of a Borel gambling problem with $u \geq 0$ is always upper analytic but need not be Borel (cf. Dubins and Sudderth [13] or Dellacherie and Meyer [9, Chapter $\mathrm{x}]$ ).

Nonnegative upper analytic functions were called "semi-analytic" by Blackwell et al. [2] and "analytique" by Dellacherie and Meyer [9]. Most of the following facts are simple variations of results in one or both of these references. All spaces considered below are Borel. The integral of a real-valued function $f$ with respect to a measure $\gamma$ will often be written as $\gamma f$.

(3.1) If $f: X \rightarrow \bar{R}$ is upper analytic and $g: Y \rightarrow X$ is Borel, then $f \circ g$ is upper analytic.

(3.2) If $f: X \rightarrow \bar{R}$ is upper analytic and $g: X \rightarrow R$ is Borel, then $f-g$ is upper analytic.

Proof. $[f-g>c]=\bigcup_{r}[f>r, g<r-c]$, where the union is over all rational numbers $r$.

(3.3) If $f, g: X \rightarrow \bar{R}$ are upper analytic and $c \in R$, then $\{x: f(x)+g(x)$ is well defined and exceeds $c$ \} is analytic.

Proof. $f(x)+g(x)$ is well defined if both $f(x)$ and $g(x)$ are greater than $-\infty$. So the set in question is just $\bigcup_{r}[f>r, g>c-r]$.

If $f_{n}: X \rightarrow \bar{R}$ are upper analytic, then so are $\inf f_{n}, \sup f_{n}$, $\liminf f_{n}$, and $\limsup f_{n}$.

If $f: X \rightarrow \bar{R}$ is upper analytic and $c \in R$, then $\{\mu \in \mathscr{P}(X)$ :

$\int f d \mu$ exists and exceeds $\left.c\right\}$ is analytic.

Proof. This is proved in [2] and in [9] for nonnegative $f$. Let $f^{+}=f \vee 0$, $f^{-}=-(f \wedge 0)$. Then $\mu \rightarrow \mu\left(f^{+}\right)$is upper analytic and $\mu \rightarrow-\mu\left(f^{-}\right)$is also. Since $\mu(f)=\mu\left(f^{+}\right)-\mu\left(f^{-}\right)$when the integral is defined, (3.3) applies.

(3.6) Suppose $K: X \times \underset{\sim}{B}(Z) \rightarrow[0,1]$ is a Borel measurable Markov kernel and $A \subseteq Y \times Z$ is analytic. Then $(x, y) \rightarrow K\left(x, A_{y}\right)$ is upper analytic.

Proof. Define $f: \mathscr{P}(Y \times Z) \rightarrow[0,1]$ by $f(\gamma)=\gamma(A)$ and $g: X \times Y \rightarrow$ $\mathscr{P}(Y \times Z)$ by $g(x, y)=\delta(y) \times K(x, \bullet)$. The function is just $f \circ g$. Apply (3.1) and (3.5). 


\section{A HIERARCHY OF RETURN FUNCTIONS}

If $t$ is a Borel stop rule and $x \in F$, define the conditional stop rule $t[x]$ to be the function on $H$ given by

$$
t[x]\left(h_{1}, h_{2}, \ldots\right)=t\left(x, h_{1}, h_{2}, \ldots\right)-1 .
$$

Then either $t[x]$ is again a stop rule or it is identically equal to zero.

There is a natural way to associate with every stop rule $t$ an ordinal number $j(t)$ called the index of $t$ by setting $j(0)=0$ and requiring

$$
j(t)=\sup \{j(t[x])+1: x \in F\} .
$$

This definition of the index is equivalent to that of Dellacherie and Meyer in [8], as was pointed out by Maitra et al. [16, Proposition 4.1]. Furthermore, $j(t)$ is familiar to students of Dubins and Savage as being the structure of the finitary function $h_{t}$ (cf. [11, $\S \S 2.7$ and 2.9]) except for the uninteresting case when $F$ is a singleton. Notice that $j(t[x])$ is always strictly less than $j(t)$.

The next lemma is a consequence of the Kunen-Martin theorem as explained by Dellacherie in the Erratum and Addendum to [4].

Lemma 4.1. If $t$ is a Borel stop rule, then $j(t)<\omega_{1}$.

By analogy with Dubins and Savage [11, p. 34], let $U_{1}=u$ and, for every ordinal $\alpha$ with $1<\alpha \leq \omega_{1}$ and $x \in F$, let

$$
U_{\alpha}(x)=\sup u(\pi)
$$

where the supremum is over policies $\pi=(\sigma, t)$ at $x$ such that $j(t)<\alpha$. Notice that $-\infty<u \leq U_{\alpha} \leq U<\infty$ for all $\alpha$.

Lemma 4.2. For every $x \in F$, there is an ordinal $\alpha<\omega_{1}$ such that $U(x)=$ $U_{\alpha}(x)$. Also, $U(x)=U_{\omega_{1}}(x)$ for all $x$.

Proof. Choose $\pi_{n}=\left(\sigma_{n}, t_{n}\right)$ at $x$ such that $U(x)=\sup u\left(\pi_{n}\right)$ and take $\alpha=$ $\sup \left(j\left(t_{n}\right)+1\right)$. The first assertion now follows easily from Lemma 4.1, as does the second.

It is natural to suspect that $U=U_{\omega}$, and this is the case when $u \geq 0$ as follows from Theorem 4.8 below. Here is a simple example where equality does not hold.

Example. Let $F=\{\ldots,-2,-1,0,1\}$; let $u(1)=-1$ and $u(x)=x$ if $x \neq 1$; let $\Gamma(x)=\{\delta(x), \delta(x+1)\}$ for $x<0, \Gamma(0)=\{\delta(0)\}, \Gamma(1)=\{\delta(1), \gamma\}$ where $\gamma u=-\infty$. It is easy to check that $U_{\omega}(1)=-1$ and $U(1)=0$.

A crucial feature of the example is the gamble $\gamma$ under which $u$ has expectation $-\infty$. Such gambles will also be crucial for the more complex Example 8.5. In that example there is, for every ordinal $\alpha<\omega_{1}$, a fortune $x$ such that $U(x)=U_{\alpha}(x)>U_{\beta}(x)$ for all $\beta<\alpha$.

The next theorem establishes the universal measurability of $U_{\alpha}$ for $\alpha<$ $\omega_{1}$. The theorem will be proved for a class of gambling problems more general than Borel problems. A problem $(F, \Gamma, u)$ is called analytic if the set 
$\Gamma=\{(x, \gamma): \gamma \in \Gamma(x)\}$ is assumed only to be an analytic subset of $F \times \mathscr{P}(F)$ rather than a Borel subset and if $u$ is assumed to be only upper analytic. Analytic problems with nonnegative utility functions were introduced by Meyer and Traki [18] and investigated by Dellacherie and Meyer [9] and by Dubins and Sudderth [13]. Blackwell et al. [2] studied an analogous class of problems in the framework of dynamic programming.

Define the operator $\Gamma^{1}$ by the formula

$$
\left(\Gamma^{1} \varphi\right)(x)=\sup _{\gamma \in \Gamma(x)} \gamma \varphi
$$

for functions $\varphi: F \rightarrow \bar{R}$ such that the integrals $\gamma \varphi$ occurring on the right are well defined.

Theorem 4.3. Let $\alpha$ be a positive ordinal less than $\omega_{1}$. Then $U_{\alpha}$ is upper analytic. If $\alpha$ is a successor ordinal and larger than 1 , then

$$
U_{\alpha}=\Gamma^{1}\left(U_{\alpha-1}\right) \text {. }
$$

If $\alpha$ is a limit ordinal, then

$$
U_{\alpha}=\sup \left\{U_{\beta}: \beta<\alpha\right\} .
$$

The proof of Theorem 4.3 will be based on a study of certain sets of probability measures defined on the Borel subsets of $H$. These sets will play a critical role in the proof of Theorem 1.2 as well.

Recall that $\Sigma(x)$ is the collection of all strategies $\sigma$, considered as probability measures on $\underset{\sim}{B}(H)$, available at $x$. Set

$$
\Sigma=\{(x, \sigma): \sigma \in \Sigma(x)\} .
$$

Lemma 4.4. If $\Gamma$ is a Borel (analytic) subset of $F \times \mathscr{P}(F)$, then $\Sigma$ is a Borel (analytic) subset of $F \times \mathscr{P}(F)$.

Proof. The Borel case is in Sudderth [23] and the analytic case is in Dellacherie [6]. (The proof in [23] can be modified to handle the analytic case as well.)

Next let $t$ be a stop rule and define a mapping $\varphi_{t}: H \rightarrow H$ by

$$
\begin{aligned}
\varphi_{t}(h)_{n} & =h_{n} & & \text { if } n \leq t(h), \\
& =h_{t(h)} & & \text { if } n>t(h) .
\end{aligned}
$$

Then, for each $\mu \in \mathscr{P}(H)$, define the measure $\mu^{t} \in \mathscr{P}(H)$ to be $\mu \varphi_{t}^{-1}$. Intuitively, $\mu^{t}$ is just the measure $\mu$ stopped at time $t$. By analogy with Dubins and Savage [11, p. 45], we say that $\mu^{t}$ is surely stagnant and stagnates by time $t$. For each $x \in F$, let $\Delta(x)$ be the measure in $\mathscr{P}(H)$ which assigns mass one to the singleton $\{(x, x, \ldots)\}$. We say that $\Delta(x)$ stagnates immediately or stagnates by time zero and set

$$
\mathscr{P}_{1}=\{\Delta(x): x \in F\} .
$$


For each ordinal $\alpha>1$, let $\mathscr{P}_{\alpha}$ be the collection of $\mu$ in $\mathscr{P}(H)$ such that $\mu$ stagnates by time $t$ for some stop rule $t$ with $j(t)<\alpha$. By Lemma 4.1, the union of all the $\mathscr{P}_{\alpha}$ is just $\mathscr{P}_{\omega_{1}}$. These collections of measures are central to the main arguments of this paper. Some of their properties are listed in the next lemma. For its statement and proof some more notation is needed.

For $\mu \in \mathscr{P}(H)$ and $x \in F$, write $\mu[x]$ for the conditional $\mu$-distribution of $\left(h_{2}, h_{3}, \ldots\right)$ given $h_{1}=x$. By Lemma 2.2 we can take $\mu[x]=\nu(\mu, x)$ to be a Borel mapping from $\mathscr{P}(H) \times F$ to $\mathscr{P}(H)$ and we will choose such a version in the proof of the lemma. If $t$ is a stop rule, it is straightforward to verify that

$$
\mu^{t}[x]=\mu[x]^{t[x]}, \quad \mu_{0} \text {-a.s. }
$$

where $\mu_{0}$ is the marginal distribution of $\mu$ on the first coordinate of $H, t[x]$ is the conditional stop rule, and we set

$$
\mu[x]^{0}=\Delta(x) .
$$

Lemma 4.5. Let $\alpha$ be a positive ordinal less than $\omega_{1}$. Then the following hold:

(i) $\mathscr{P}_{\alpha}$ is a Borel subset of $\mathscr{P}(H)$.

(ii) If $\alpha>1$ is not a limit ordinal, then $\mu \in \mathscr{P}_{\alpha}$ if and only if

$$
\mu_{0}\left\{x: \mu[x] \in \mathscr{P}_{\alpha-1}\right\}=1 .
$$

(iii) If $\alpha$ is a limit ordinal, then

$$
\mathscr{P}_{\alpha}=\bigcup\left\{\mathscr{P}_{\beta}: \beta<\alpha\right\} \text {. }
$$

(iv) There is a Borel mapping $\tau_{\alpha}: \mathscr{P}_{\alpha} \times H \rightarrow N$ such that $\tau_{\alpha}(\mu)=\tau_{\alpha}(\mu, \bullet)$ is a stop rule of index less than $\alpha$ or is identically zero and $\mu$ stagnates by time $\tau_{\alpha}(\mu)$.

Proof. The proof is by induction on $\alpha . \mathscr{P}_{1}$ is closed and we can take $\tau_{1}$ identically equal to zero to see that the conditions are satisfied for $\alpha=1$. So assume $\alpha>1$, and that (i) through (iv) hold for $\beta<\alpha$.

Suppose first that $\alpha$ is not a limit ordinal. Consider (ii). If $\mu \in \mathscr{P}_{\alpha}$, formula (4.6) follows from the remarks preceding the lemma. If (4.6) holds, define

$$
t(h)= \begin{cases}\tau_{\alpha-1}\left(\mu\left[h_{1}\right],\left(h_{2}, h_{3}, \ldots\right)\right)+1 & \text { if } \mu\left[h_{1}\right] \in \mathscr{P}_{\alpha-1}, \\ 1 & \text { if not. }\end{cases}
$$

Then $\mu$ stagnates by time $t$ and $j(t)<\alpha$. So $\mu \in \mathscr{P}_{\alpha}$. Thus (ii) holds. Condition (i) follows from (ii) and Lemma 2.1. For (iv), define $\tau_{\alpha}(\mu, h)$ by the formula first used for $t(h)$.

Assume now that $\alpha$ is a limit ordinal. Condition (iii) is obvious and then (i) is too. For (iv), define $\tau_{\alpha}(\mu, h)$ to be $\tau_{\beta}(\mu, h)$ where $\beta$ is the least ordinal such that $\mu$ belongs to $\mathscr{P}_{\beta}$.

We are almost ready to complete the proof of Theorem 4.3. First notice that, if we define $u^{*}: H \rightarrow R$ by

$$
u^{*}(h)=\lim _{n} \sup u\left(h_{n}\right)
$$


then, for $x \in F$,

$$
U_{\alpha}(x)=\sup \left\{\sigma u^{*}: \sigma \in \mathscr{P}_{\alpha} \cap \Sigma(x)\right\}
$$

This is because, for a policy $\pi=(\sigma, t)$ available at $x$,

$$
u(\pi)=\int u\left(h_{t}\right) d \sigma=\int u^{*} d \sigma^{t}
$$

(Notice that the integrals $\sigma u^{*}$ occurring in (4.8) are well defined because of our assumption that $u(\pi)$ is.) Thus the supremum in (4.8) is the same as that in the definition of $U_{\alpha}$.

Proof of Theorem 4.3. Each $U_{\alpha}$ is upper analytic because for every real $c$, the set $\left\{x: U_{\alpha}(x)>c\right\}$ is, by (4.8), the projection of $\left\{(x, \sigma): \sigma \in \mathscr{P}_{\alpha} \cap \Sigma(x)\right.$, $\left.\sigma u^{*}>c\right\}$. The latter set is analytic, as follows from (3.4), (3.5), and Lemmas 4.4 and 4.5.

Suppose next that $\alpha$ is a successor ordinal. Fix $x_{0}$ and $\gamma \in \Gamma\left(x_{0}\right)$. Because $U_{\alpha-1}$ is universally measurable, there is a Borel function $Q: F \rightarrow[-\infty, \infty)$ such that $Q \leq U_{\alpha-1}$ and $Q=U_{\alpha-1} \quad \gamma$-almost surely. Let $\varepsilon>0$ and

$$
E=\left\{(x, \sigma): \sigma \in \mathscr{P}_{\alpha-1} \cap \Sigma(x), \sigma u^{*}>Q(x)-\varepsilon\right\} .
$$

Then $E$ is an analytic set with nonempty $x$-sections. So, by the von Neumann selection theorem (cf. [2] or [19, 4E.9, p. 240]), there is a universally measurable function $\bar{\sigma}: F \rightarrow \mathscr{P}(H)$ such that $(x, \bar{\sigma}(x)) \in E$ for all $x$. Choose a Borel set $B \subseteq F$ such that $\gamma(B)=1$ and $\bar{\sigma}$ restricted to $B$ is Borel. Define a measure $\hat{\sigma}$ in $\mathscr{P}(H)$ to have marginal $\hat{\sigma}_{0}=\gamma$ on the first coordinate and conditional distribution

$$
\begin{aligned}
\hat{\sigma}[x]=\bar{\sigma}(x) & \text { if } x \in B, \\
& =\Delta(x) \quad \text { if } x \notin B .
\end{aligned}
$$

Then $\hat{\sigma} \in \mathscr{P}_{\alpha} \cap \Sigma\left(x_{0}\right)$ by Lemma $4.5($ ii) and, hence,

$$
\begin{aligned}
\infty & >U_{\alpha}\left(x_{0}\right) \geq \hat{\sigma} u^{*}=\int\left(\bar{\sigma}(x) u^{*}\right) \gamma(d x) \\
& \geq \int Q d \gamma-\varepsilon=\int U_{\alpha-1} d \gamma-\varepsilon .
\end{aligned}
$$

Notice that this calculation shows that the integral $\gamma U_{\alpha-1}$ is well defined for every $\gamma \in \Gamma\left(x_{0}\right)$. Take the supremum over $\gamma$ and let $\varepsilon \rightarrow 0$ to get

$$
U_{\alpha}\left(x_{0}\right) \geq \Gamma^{1}\left(U_{\alpha-1}\right)\left(x_{0}\right) \text {. }
$$

For the opposite inequality, let $\sigma \in \mathscr{P}_{\alpha} \cap \Sigma\left(x_{0}\right)$. Then

$$
\sigma u^{*}=\int\left(\sigma[x] u^{*}\right) \sigma_{0}(d x) \leq \int U_{\alpha-1} d \sigma_{0},
$$

where the equality is by conditioning on $h_{1}=x$ and the fact that $u^{*}$ is shift invariant and the inequality is by (4.6). Take the supremum over $\sigma$ to get

$$
U_{\alpha}\left(x_{0}\right) \leq \Gamma^{1}\left(U_{\alpha-1}\right)\left(x_{0}\right) \text {. }
$$


The equality (4.3) is obvious. The proof of Theorem 4.3 is now complete. The next two lemmas will enable us to prove a generalization of Theorem 1.1.

Lemma 4.6. Let $\pi=(\sigma, t)$ be a policy for which $u(\pi)<\infty$ and for which the process $\left\{u^{-}\left(h_{n}\right)\right\}$ is uniformly integrable under $\sigma$. Then, for every stop rule $t$, $u(\sigma, t) \leq \sup _{n} u(\sigma, t \wedge n)$.

Proof. Calculate as follows:

$$
\begin{aligned}
u(\sigma, t)-u(\sigma, t \wedge n) & =\int_{[t>n]} u\left(h_{t}\right) d \sigma-\int_{[t>n]} u\left(h_{n}\right) d \sigma \\
& \leq \int_{[t>n]} u^{+}\left(h_{t}\right) d \sigma+\int_{[t>n]} u^{-}\left(h_{n}\right) d \sigma .
\end{aligned}
$$

The two terms in the final expression approach zero as $n \rightarrow \infty$, the first by the dominated convergence theorem and the second by the uniform integrability of $\left\{u^{-}\left(h_{n}\right)\right\}$.

The next result is a descendant of Theorem 2.12.1 of [11].

Proposition 4.7. Suppose $Q$ is universally measurable, $Q \geq u$, and $Q$ is excessive. Then $Q \geq U$.

Proof. Let $x_{0} \in F$ and $\pi=(\sigma, t)$ be a policy at $x_{0}$ such that

$$
u(\pi)=\int u\left(h_{t}\right) d \sigma>-\infty .
$$

Because $Q \geq u, Q(\pi)$ is well defined and

$$
Q(\pi) \geq u(\pi) .
$$

Furthermore,

$$
Q\left(x_{0}\right) \geq Q(\pi)
$$

by an optional sampling theorem for everywhere finite stop rules, which can be proved, as in [11], by induction on $j(t)$. To start the induction, notice that, by Fubini,

$$
Q(\pi)=\int Q(\sigma[x], t[x]) \sigma_{0}(d x) .
$$

Now use (4.9) and (4.10), and take the supremum over $\pi$ at $x_{0}$ to get $Q\left(x_{0}\right) \geq$ $U\left(x_{0}\right)$.

Definition 4.8. The problem $(F, \Gamma, u)$ is uniformly integrable from below if, given $x \in F$ and $\varepsilon>0$, there exists a policy $\pi=(\sigma, t)$ at $x$ such that $u(\pi)>U(x)-\varepsilon$ and $\left\{u^{-}\left(h_{n}\right)\right\}$ is uniformly integrable under $\sigma$.

Theorem 4.8. If $(F, \Gamma, u)$ is uniformly integrable from below, then $U=U_{\omega}$ and $U$ is the reduced function of $u$.

Proof. It follows from Lemma 4.6 that $U(x)$ is the supremum of $u(\pi)$ taken over policies $\pi$ at $x$ with bounded stop rules. Since every stop rule bounded by 
an integer $n$ has index bounded by $n$, it then follows that $U=\sup U_{n}=U_{\omega}$. By Theorem 4.3, $U$ is upper analytic and therefore universally measurable. Obviously, $U \geq u$. Also, $U$ is excessive because, given $x$ and $\gamma \in \Gamma(x)$,

$$
\gamma U=\gamma U_{\omega} \leq \Gamma^{1}\left(U_{\omega}\right)(x)=U_{\omega+1}(x)=U(x) .
$$

Apply Proposition 4.7 to complete the proof.

\section{A THEOREM OF MoschovaKis}

The proofs in the next two sections depend on a result from the theory of inductive definability. A few definitions are needed to formulate the result.

Say that $\Phi$ is a monotone operator on a set $Z$ if $\Phi$ maps subsets of $Z$ to subsets of $Z$ and if $\Phi\left(E_{1}\right) \subseteq \Phi\left(E_{2}\right)$ whenever $E_{1} \subseteq E_{2} \subseteq Z$. Define the iterates of such a $\Phi$ by transfinite induction as follows:

$$
\begin{array}{rlrl}
\Phi^{0} & =\varnothing, & \\
\Phi^{\xi+1} & =\Phi\left(\Phi^{\xi}\right) & & \text { for successor ordinals } \xi+1, \\
\Phi^{\xi} & =\bigcup_{\eta<\xi} \Phi^{\eta} & & \text { for limit ordinals } \xi .
\end{array}
$$

It is easy to verify that $\Phi^{\infty}$, the least fuxed point of $\Phi$, is given by $\bigcup\left\{\Phi^{\xi}: \xi<\right.$ $\kappa$, where $\kappa$ is the least cardinal greater than the cardinality of $Z$.

Theorem 5.1. Suppose $Z$ is a Borel set and let $\Phi$ be a monotone operator on $Z$. Assume that, for any Polish space $Y$ and any coanalytic set $C \subseteq Y \times Z$, the set

$$
C^{*}=\left\{(y, z) \in Y \times Z: z \in \Phi\left(C_{y}\right)\right\}
$$

is coanalytic, where $C_{y}$ is the $y$-section of $C$. Then

(a) $\Phi^{\infty}$ is a coanalytic subset of $Z$;

(b) $\Phi^{\infty}=\Phi^{\omega_{1}}$;

(c) if $A$ is an analytic subset of $\Phi^{\infty}$, there is an $\eta<\omega_{1}$ such that $A \subseteq \Phi^{\eta}$.

Part (a) of the theorem is a special case of a very general result of Moschovakis [19, 7C.8, p. 414]. Parts (b) and (c) are not stated explicitly in [19], but they can be deduced from results there and this deduction is carried out by Louveau [15]. A related result is in Dellacherie [5].

\section{The SET $\mathscr{P}_{\omega_{1}}$ AND the UNIVERSAL MEASURABILITY OF $U$}

Theorem 5.1 will be used in this section to show

Theorem 6.1. The set $\mathscr{P}_{\omega_{1}}$ of surely stagnant measures is coanalytic.

It can also be shown that, if $F$ is infinite, then $\mathscr{P}_{\omega_{1}}$ is not a Borel set. However, we will be content to point out in $\S 8$ that $\mathscr{P}_{\omega_{1}}{ }_{1}$ is not Borel when $F$ is an uncountable Borel set. 
After the proof of Theorem 6.1, the proof of Theorem 1.2(i) will be given. Proof of Theorem 6.1. To apply Theorem 5.1, take $Z=\mathscr{P}(H)$ and, for $E \subseteq Z$, define

$$
\Phi(E)=\mathscr{P}_{1} \cup\left\{\mu: \mu_{0}^{*}\{x: \mu[x] \in E\}=1\right\}
$$

where $\mathscr{P}_{1}$, as in (4.5), is the collection of point-masses $\Delta(x), \mu_{0}$ is the marginal of $\mu$ on the first coordinate of $H, \mu_{0}^{*}$ is the outer measure associated with $\mu_{0}$, and $\mu[x]$ is, as in Lemma 2.2, a Borel version of the conditional $\mu$-distribution of $\left(h_{2}, h_{3}, \ldots\right)$ given $h_{1}=x$. Clearly, $\Phi$ is monotone.

To verify the remaining hypothesis of Theorem 5.1, let $Y$ be a Polish space and let $C \subseteq Y \times Z$ be coanalytic. The set

$$
\bar{C}=\{(y, \mu, x) \in Y \times Z \times F:(y, \mu[x]) \in C\}
$$

is also coanalytic since it is the inverse image of $C$ under the Borel mapping $(y, \mu, x) \rightarrow(y, \mu[x])$. Define a Borel Markov kernel $K$ on $(Y \times Z) \times \underset{\sim}{B}(F)$ by

$$
K((y, \mu), B)=\mu_{0}(B) .
$$

Apply (3.6) to see that

$$
C^{*}=\left\{(y, \mu) \in Y \times Z: \mu \in \mathscr{P}_{1} \text { or } K\left((y, \mu), \bar{C}_{(y, \mu)}\right)=1\right\}
$$

is coanalytic.

Thus Theorem 5.1 applies to show that

$$
\Phi^{\infty}=\Phi^{\omega_{1}}=\bigcup_{\eta<\omega_{1}} \Phi^{\eta}
$$

is coanalytic. Now, by definition,

$$
\mathscr{P}_{\omega_{1}}=\bigcup_{\eta<\omega_{1}} \mathscr{P}_{\eta} .
$$

So the following lemma will complete the proof.

Lemma 6.2. For $0<\eta<\omega_{1}, \Phi^{\eta}=\mathscr{P}_{\eta}$ and, hence, $\Phi^{\omega_{1}}=\mathscr{P}_{\omega_{1}}$.

Proof. By definition, $\Phi^{1}=\Phi(\varnothing)=\mathscr{P}_{1}$. Let $1<\xi<\omega_{1}$ and assume $\Phi^{\eta}=\mathscr{P}_{\eta}$ for $0<\eta<\xi$. Use (5.1), (6.1), and Lemma 4.5(ii), (iii) to see that $\Phi^{\xi}=$ $\mathscr{P}_{\xi}$.

Here is a generalization of Theorem 1.2(i) which includes analytic problems and does not assume $u$ is nonpositive. The definition of the reduced function is the same for an analytic problem as for a Borel problem.

Theorem 6.3. For each $a \in R$, the set $[U>a]$ is a PCA set. If all PCA sets are universally measurable, then $U$ is the reduced function of $u$.

Proof. The proof is analogous to that of Theorem 4.3 and will use some of the same notation. 
For $a \in R$, define the set

$$
E=\left\{(x, \sigma): \sigma \in \Sigma(x) \cap \mathscr{P}_{\omega_{1}}, \sigma u^{*}>a\right\} .
$$

This is a PCA set as follows from Lemma 4.4, Theorem 6.1, (3.4), and (3.5). Now, for each $x$,

$$
U(x)=\sup \left\{\sigma u^{*}: \sigma \in \Sigma(x) \cap \mathscr{P}_{\omega_{1}}\right\}
$$

Hence, $\{x: U(x)>a\}$ is the projection of $E$ onto the first coordinate and is therefore a PCA set also.

Suppose now that all PCA sets are universally measurable and, hence, that $U$ is universally measurable. As is always the case, $U \geq u$. So, by Proposition 4.7 , it remains to be shown only that $U$ is excessive.

Let $x_{0} \in F, \gamma \in \Gamma\left(x_{0}\right)$, and $\varepsilon>0$. It suffices to show that $\gamma U \leq U\left(x_{0}\right)+\varepsilon$. Let $Q: F \rightarrow[-\infty, \infty)$ be a Borel measurable function such that $Q \leq U$ and $Q=U \quad \gamma$-almost surely. Let

$$
C=\left\{(x, \sigma): \sigma \in \Sigma(x) \cap \mathscr{P}_{\omega_{1}}, \sigma u^{*}>Q(x)-\varepsilon\right\} .
$$

Then $C$ is a PCA set for the same reasons that $E$ is. As an easy consequence of the Novikov-Kondo-Addison theorem [19, 4E.4, p. 235], there is a function $\bar{\sigma}: F \rightarrow \mathscr{P}(H)$ such that $(x, \bar{\sigma}(x)) \in C$ for all $x$ and the graph $G$ of $\bar{\sigma}$ is PCA. It follows that $\bar{\sigma}$ is universally measurable because, given a Borel set $B \subset \mathscr{P}(H)$, the set $\bar{\sigma}^{-1}(B)$ is the projection onto $F$ of the PCA set $G \cap(F \times B)$ and, hence, is itself a PCA set.

Choose a Borel set $B \subset F$ such that $\gamma(B)=1$ and $\bar{\sigma}$ restricted to $B$ is Borel. Define $\hat{\sigma} \in \mathscr{P}(H)$ by setting $\hat{\sigma}_{0}=\gamma$ and

$$
\begin{aligned}
& \hat{\sigma}[x]=\bar{\sigma}(x) \quad \text { if } x \in B, \\
& =\Delta(x) \text { if } x \in B \text {. }
\end{aligned}
$$

Then $\hat{\sigma} \in \Sigma\left(x_{0}\right)$.

Next $\bar{\sigma}(B)$ is an analytic subset of $\mathscr{P}_{\omega_{1}}$. So, by Theorem 5.1(c) and Lemma 6.2 , there is a $\xi<\omega_{1}$ such that $\bar{\sigma}(B) \subset \mathscr{P}_{\xi}$. Hence, $\hat{\sigma}[x] \in \mathscr{P}_{\xi}$ for all $x$, and, by Lemma 4.5 (ii),

$$
\hat{\sigma} \in \mathscr{P}_{\xi+1} \cap \Sigma\left(x_{0}\right)
$$

Hence,

$$
\infty>U\left(x_{0}\right) \geq \hat{\sigma} u^{*}=\int\left(\hat{\sigma}[x] u^{*}\right) \gamma(d x) \geq \int Q d \gamma-\varepsilon=\int U d \gamma-\varepsilon .
$$

\section{COUNTABLE HOUSES (INCLUdING STOP-OR-GO PROBLEMS)}

The result of this section is the following generalization of the first two assertions of Theorem 1.3. 
Theorem 7.1. Assume $(F, \Gamma, u)$ is a Borel problem and that $\Gamma(x)$ is countable for every $x$. Then (i) the function $-U$ is upper analytic and $U$ is the reduced function of $u$, and (ii) if $(F, \Gamma, u)$ is uniformly integrable from below, $U$ is Borel.

Under our hypotheses, $\Gamma$ is a Borel subset of $F \times \mathscr{P}(F)$ with countable $x$-sections. By a theorem of Luzin (cf. Moschovakis $[19,4 \mathrm{~F} .17$, p. 259]) there exist Borel mappings $\gamma_{1}, \gamma_{2}, \ldots$ from $F$ to $\mathscr{P}(F)$ such that

$$
\Gamma(x)=\left\{\gamma_{1}(x), \gamma_{2}(x), \ldots\right\}
$$

for every $x \in F$.

The proof of (ii) is now quite easy. Since $U_{1}=u$ is Borel and, by Theorem 4.3 , for every $x$,

$$
U_{n+1}(x)=\sup _{m} \gamma_{m}(x) U_{n},
$$

it follows that $U_{2}, U_{3}, \ldots$ are Borel. So $U_{\omega}=\sup U_{n}$ is also Borel. But $U=U_{\omega}$ by Theorem 4.8. (The special case of (ii) when $u$ is bounded was proved in [23].)

The rest of the section is devoted to proving (i). By Proposition 4.7, we need only show that $U$ is universally measurable and excessive. The key idea of the proof is another application of Theorem 5.1. We will first define the operator $\Phi$ and then explain its connection to our situation.

Let $Z=F \times R$ and, for $E \subseteq Z$, define $\Phi(E)$ to be the set

$$
\begin{aligned}
\left\{(x, a) \in Z: \max \left\{u(x), \sup _{n}\left(\left(\gamma_{n}(x)\right.\right.\right.\right. & \times \lambda)^{*}\left(E \cap Z^{+}\right) \\
& \left.\left.\left.-\left(\gamma_{n}(x) \times \lambda\right)^{*}\left(E^{c} \cap Z^{-}\right)\right)\right\}>a\right\},
\end{aligned}
$$

where $Z^{+}=\{(x, a) \in Z, a \geq 0\}, Z^{-}=\{(x, a) \in Z: a \leq 0\}, \lambda$ is Lebesgue measure on $R, \gamma_{n}(x) \times \lambda$ is the product measure on $Z$, and $\mu^{*}$ denotes the outer measure induced by a measure $\mu$. The convention is also made that $\infty-\infty=\infty$ in the expression (7.1). To understand the operator $\Phi$, consider the special case

$$
E=\{(x, a): v(x)>a\}
$$

where $v: F \rightarrow R$ is universally measurable and such that the integrals $\gamma_{n}(x) v$ are well defined for every $x \in F$ and $n \in N$. Then

$$
\begin{aligned}
\left(\gamma_{n}(x) \times \lambda\right)\left(E \cap Z^{+}\right) & =\int \lambda\left(\left(E \cap Z^{+}\right)_{y}\right) \gamma_{n}(x)(d y) \\
& =\int v^{+}(y) \gamma_{n}(x)(d y)
\end{aligned}
$$

and

$$
\begin{aligned}
\left(\gamma_{n}(x) \times \lambda\right)\left(E^{c} \cap Z^{-}\right) & =\int \lambda\left(\left(E^{c} \cap Z^{-}\right)_{y}\right) \gamma_{n}(x)(d y) \\
& =\int v^{-}(y) \gamma_{n}(x)(d y) .
\end{aligned}
$$


Thus the difference of (7.2) and (7.3) is just the integral $\gamma_{n}(x) v$ and, in this special case, the set $\Phi(E)$ of $(7.1)$ is just

$$
\left\{(x, a) \in Z: \max \left\{u(x), \Gamma^{1} v(x)\right\}>a\right\} .
$$

Lemma 7.3. For $1 \leq \xi \leq \omega_{1}, \Phi^{\xi}=\left\{(x, a): U_{\xi}(x)>a\right\}$.

Proof. Use Theorem 4.3 and the definition of $\Phi^{\xi}$ in (5.1).

Suppose, for a moment, that the hypotheses of Theorem 5.1 are satisfied. Then, by that theorem and Lemmas 4.2 and 7.3,

$$
\Phi^{\infty}=\Phi^{\omega_{1}}=\left\{(x, a): U_{\omega_{1}}(x)>a\right\}=\{(x, a): U(x)>a\}
$$

is coanalytic, and, consequently, for each $a$,

$$
\Phi_{a}^{\infty}=\{x: U(x)>a\}
$$

is coanalytic too. In particular, $-U$ is upper analytic.

Also, by Theorem 5.1, $\Phi^{\omega_{1}}$ is a fixed point of $\Phi$ and, hence,

$$
\{(x, a): U(x)>a\}=\left\{(x, a): \Gamma^{1} U(x)>a\right\} .
$$

Thus $\Gamma^{1} U=U$ and, in particular, $U$ is excessive.

Thus we need only verify the hypotheses of Theorem 5.1. Clearly, $\Phi$ is monotone. So let $Y$ be Polish and $C \subset Y \times Z$ be coanalytic. We must check that the set $C^{*}$ of $(5.2)$ is coanalytic.

First, for each pair of positive integers $m$ and $n$, let $\lambda_{n}$ be Lebesgue measure on the interval $I_{n}=[n-1, n]$ and define the mapping $\varphi_{m, n}: Y \times F \rightarrow[0,1]$ by

$$
\varphi_{m, n}(y, x)=\left(\gamma_{m}(x) \times \lambda_{n}\right)\left(C_{y}^{c} \cap\left(F \times I_{n}\right)\right) .
$$

This mapping is upper analytic, by (3.1) and (3.6), since it equals

$$
K\left(\gamma_{m}(x),\left(C^{c} \cap\left(Y \times F \times I_{n}\right)\right)_{y}\right)
$$

where $K$ is the Borel kernel on $\mathscr{P}(F) \times \underset{\sim}{B}\left(F \times I_{n}\right)$ defined by

$$
K(\gamma, B)=\left(\gamma \times \lambda_{n}\right)(B) .
$$

Hence,

$$
\begin{aligned}
-\left(\gamma_{m}(x) \times \lambda\right)\left(C_{y} \cap Z^{+}\right) & =-\sum_{n}\left(\gamma_{m}(x) \times \lambda_{n}\right)\left(C_{y} \cap\left(F \times I_{n}\right)\right) \\
& =\sum_{n}\left(\varphi_{m, n}(y, x)-1\right)
\end{aligned}
$$

is an upper analytic function of $(y, x)$ by (3.3) and (3.4). Similarly,

$$
\left(\gamma_{m}(x) \times \lambda\right)\left(C_{y}^{c} \cap Z^{-}\right)
$$

is an upper analytic function of $(y, x)$. Finally,

$$
\begin{aligned}
C^{*}=\left\{(y, x, a): \max \left\{u(x), \sup _{m}\left(\left(\gamma_{m}(x) \times \lambda\right)\left(C_{y} \cap Z^{+}\right)\right.\right.\right. \\
\left.\left.\left.-\left(\gamma_{m}(x) \times \lambda\right)\left(C_{y}^{c} \cap Z^{-}\right)\right)\right\}>a\right\}
\end{aligned}
$$


is coanalytic by (3.2), a variant of (3.3), (3.4), and our hypothesis that $u$ is Borel.

The proof of Theorem 7.1 is now complete.

\section{Counterexamples}

An example is presented in this section which, along with several modifications, will establish all the negative results of the paper such as Theorem 1.2(ii). This example is based on a result of Dellacherie and Meyer [8] and is a close relative of an example of Blackwell and Ramakrishnan [3].

Let $N=\{1,2, \ldots\}$ and let $T$ be the collection of all stopping times on $N^{N}$; i.e., $T$ is the set of mappings $t: N^{N} \rightarrow N \cup\{\infty\}$ such that, for every $n \in N$, the set $[t \leq n]$ is measurable with respect to the sigma-field generated by the first $n$ coordinate mappings on $N^{N}$. Give $N \cup\{\infty\}$ the usual compact topology and give $T$ its topology of pointwise convergence. Then $T$ is a compact metric space.

Let $S$ be the set of all (complete) stop rules in $T$; i.e.,

$$
S=\left\{t \in T: t(y)<\infty \text { for all } y \in N^{N}\right\} .
$$

The following nice result of Dellacherie and Meyer [8, Theorem 2] is the key to our example.

Lemma 8.1. The set $S$ is coanalytic and is not analytic.

Here is the basic example.

Example 8.2. Define $\bar{T}=T \cup\{0\}$ and $\bar{N}=N \cup\{0\}$. Then let $F=\bar{T} \times N \times \bar{N}$ and, for $x=(t, n, m) \in F$, define $u(x)=-m$. The gambling house $\Gamma$ will be a stop-or-go house so that $\Gamma(x)=\{\gamma(x), \delta(x)\}$ for every $x$, where $\gamma(x)$ is defined as follows: If $x=(0, n, m)$, then $\gamma(x)=\delta(0, n, 0)$. If $x=(t, n, m)$ and $t \neq 0$, then, for every $k \in N, \gamma(x)$ assigns probability $2^{-k}$ to the fortune $\left(t[k], k, 2^{k} m\right)$. (Here $t[k]$ is the conditional stop rule $t[k]\left(n_{1}, n_{2}, \ldots\right)=t\left(k, n_{1}, n_{2}, \ldots\right)-1$, or is the element 0 of $\bar{T}$.) It is not difficult to verify that $(F, \Gamma, u)$ is a Borel problem.

Lemma 8.3. The optimal return function $U$ is not upper analytic and, therefore, not Borel. Indeed $U(t, 1,1)=0$ if $t \in S$ and $U(t, 1,1)=-1$ if $t \in T \backslash S$.

Proof. By Lemma 8.1, it suffices to prove the final assertion.

Let $\sigma$ be the strategy at $(t, 1,1)$ which always uses $\gamma(x)$ at any fortune $x$. First assume $t \in S$ and define a stop rule $\tau$ on $H=F^{N}$ by

$$
\tau\left(\left(t_{1}, n_{1}, m_{1}\right),\left(t_{2}, n_{2}, m_{2}\right), \ldots\right)=t\left(n_{1}, n_{2}, \ldots\right) .
$$

Now $\sigma$ assigns probability one to histories $h$ of the form

$$
\begin{aligned}
& \left(\left(t\left[n_{1}\right], n_{1}, 2^{n_{1}}\right),\left(t\left[n_{1}\right]\left[n_{2}\right], n_{2}, 2^{n_{1}+n_{2}}\right), \ldots,\left(0, n_{k}, 2^{n_{1}+\ldots+n_{k}}\right),\right. \\
& \left.\left(0, n_{k}, 0\right),\left(0, n_{k}, 0\right), \ldots\right)
\end{aligned}
$$


where 0 occurs in the first coordinate of $h_{k}$ at time $\tau(h)=t\left(n_{1}, n_{2}, \ldots\right)$ and 0 occurs in the third coordinate of $h_{k}$ for all $k \geq \tau(h)+1$. Hence,

$$
u\left(h_{\tau+1}\right)=0, \quad \sigma \text {-almost surely, }
$$

and

$$
U(t, 1,1) \geq \int u\left(h_{\tau+1}\right) d \sigma=0 .
$$

Obviously, $U(t, 1,1) \leq 0$ since $u(x) \leq 0$ for all $x$.

Next suppose $t \notin S$ so that there is a sequence $\left(n_{1}, n_{2}, \ldots\right) \in N^{N}$ such that $t\left(n_{1}, n_{2}, \ldots\right)=\infty$. Let $\tau$ be any (everywhere finite) stop rule on $H$ and let

$$
h^{*}=\left(\left(t\left[n_{1}\right], n_{1}, 2^{n_{1}}\right),\left(t\left[n_{1}\right]\left[n_{2}\right], n_{2}, 2^{n_{1}+n_{2}}\right), \ldots\right) .
$$

Suppose $\tau\left(h^{*}\right)=k$. Then

$$
\begin{aligned}
\int u\left(h_{\tau}\right) d \sigma & \leq u\left(h_{\tau}^{*}\right) \sigma\left\{h: h_{1}=h_{1}^{*}, \ldots, h_{k}=h_{k}^{*}\right\} \\
& =-2^{n_{1}+\cdots+n_{k}} 2^{-\left(n_{1}+\cdots+n_{k}\right)} \\
& =-1 .
\end{aligned}
$$

A similar calculation will show the return from any policy at $(t, 1,1)$ is no larger than -1 . Of course, $U(t, 1,1) \geq u(t, 1,1)=-1$. So $U(t, 1,1)=$ -1 .

The final assertion of Theorem 1.3 is immediate from the lemma, and the proof of that theorem is now complete. We have also established with this example that the statement from Theorem 7.1 that $-U$ is upper analytic cannot be improved.

Corollary. The set $\mathscr{P}_{\omega_{1}}$ is not Borel.

Proof. If $\mathscr{P}_{\omega_{1}}$ were Borel, it would follow from (6.2) that $U$ is upper analytic.

Here is our first modification of Example 8.2.

Example 8.4. Let $(F, \Gamma, u)$ be as in Example 8.2. Let $X$ be a Polish space disjoint from $F$ and $A$ an analytic subset of $X$. Set $F_{1}=F \cup X$ and give $F_{1}$ the union topology which is the union of the topologies on $F$ and $X$. We will define $u_{1}$ and $\Gamma_{1}$ so that the optimal return $U^{1}$ of $\left(F_{1}, \Gamma_{1}, u_{1}\right)$ satisfies

$$
\left\{x \in X: U^{1}(x)=0\right\}=\left\{x \in X: U^{1}(x) \geq 0\right\}=X \backslash A .
$$

Take the restrictions of $u_{1}$ and $\Gamma_{1}$ to $F$ to be $u$ and $\Gamma$, respectively, and set $u_{1}(x)=-1$ for $x \in X$. To define $\Gamma_{1}$ on $X$, first write

$$
A=\bigcup_{y} \bigcap_{k} C(y \mid k)
$$


where $y=\left(y_{1}, y_{2}, \ldots\right)$ ranges over $N^{N}$ and, for each $y \in N^{N}$ and $k \in N$, $C(y \mid k)=C\left(y_{1}, \ldots, y_{k}\right)$ is a closed subset of $X$ and $C(y \mid k) \supseteq C(y \mid k+1)$. The proof that such sets exist can be found, for example, in [14] or [8].

Next define a mapping $\psi: X \rightarrow F$ as follows: for $x \in X$, let

$$
\psi(x)=\left(t_{x}, 1,1\right)
$$

where $t_{x} \in T$ is given by

$$
t_{x}(y)=\inf \{k: x \notin C(y \mid k)\}
$$

for each $y \in N^{N}$. (The mapping $t_{x}$ was introduced and its properties were studied by Dellacherie and Meyer [8, p. 375].) Check that $\psi$ is Borel because $\left\{x: t_{x}(y) \leq k\right\}$ is open for fixed $y \in N^{N}$ and $k \in N$. Then notice that $t_{x}(y)$ is finite for every $y$ if and only if $x \notin A$ so that

$$
X \backslash A=\left\{x: t_{x} \in S\right\}=\psi^{-1}\{(t, 1,1): t \in S\} .
$$

Finally, define

$$
\Gamma_{1}(x)=\{\delta(x), \delta(\psi(x))\}
$$

for $x \in X$. Then $\Gamma_{1}$ is Borel and, for $x \in X, U^{1}(x)=U(\psi(x))=0$ if and only if $t_{x} \in S$. Thus (8.1) holds.

Here is our second modification of the basic example.

Example 8.5. Begin with Example 8.4 but take the space $X=I \times I$ where $I=[0,1]$ is a copy of the unit interval disjoint from $F_{1}$. Set $F_{2}=F_{1} \cup I=$ $F \cup(I \times I) \cup I$ with the union topology. Let $u_{2}$ and $\Gamma_{2}$ restricted to $F_{1}$ be $u_{1}$ and $\Gamma_{1}$, respectively, and, for $x_{1} \in I$, define

$$
u_{2}\left(x_{1}\right)=-1, \quad \Gamma_{2}\left(x_{1}\right)=\left\{\delta\left(x_{1}\right)\right\} \cup\left\{\delta\left(x_{1}, x_{2}\right): x_{2} \in I\right\} .
$$

If $U^{2}$ is the optimal return function for the Borel problem $\left(F_{2}, \Gamma_{2}, u_{2}\right)$ and $P$ is the PCA set given by

$$
P=\left\{x_{1} \in I:\left(\exists x_{2} \in I\right)\left(\left(x_{1}, x_{2}\right) \in X \backslash A\right)\right\},
$$

Then, clearly,

$$
\left\{x_{1} \in I: U^{2}\left(x_{1}\right)=0\right\}=P .
$$

Thus $U^{2}$ is universally measurable only if $P$ is. (Indeed, $U^{2}\left(x_{1}\right)=-1$ if $x_{1} \in I \backslash P$. So $U^{2}$ is universally measurable if $P$ is.)

The first assertion of Theorem 1.2(ii) is now proved. To establish the second assertion, define, for each $z \in I \backslash P$, the function $Q_{z}: F_{2} \rightarrow R$ by

$$
\begin{aligned}
Q_{z}(x) & =-1 & & \text { if } x=z, \\
& =0 & & \text { if } x \in I, x \neq z, \\
& =U^{2}(x) & & \text { if } x \in F_{1} .
\end{aligned}
$$

For each $z \in I \backslash P, Q_{z} \geq u_{2}$ and $Q_{z}$ is excessive and universally measurable. This is trivial to verify on $I$ and follows on $F_{1}$ from Theorem 1.3 applied to 
the Borel stop-or-go problem $\left(F_{1}, \Gamma_{1}, u_{1}\right)$. By Proposition 4.7, if there is a reduced function $R u_{2}$, it must satisfy

$$
Q_{z} \geq R u_{2} \geq U^{2}
$$

for every $z \in I \backslash P$. But inf $Q_{z}=U^{2}$. Thus $U^{2}$ is the only possible candidate. However, $U^{2}$ is not measurable unless $P$ is, as was already pointed out. The proof of Theorem 1.2 is now complete.

The first result on the measurability of $U$ was proved by Dubins and Savage under the assumptions that $F$ is a compact metric space and that $u$ and $\Gamma$ satisfy certain continuity requirements [11, Theorem 2.16.1]. It is easy to alter Example 8.5 so that all the assumptions of Dubins and Savage are satisfied with the exception of the compactness of $F$. To carry out such an alteration, just replace the unit interval $I$ by the Cantor space $2^{\omega}$ of all sequences of 0 's and 1's. Because the clopen sets of $2^{\omega}$ form a basis for its topology, the sets $C(y \mid k)$ in Example 8.4 can be taken to be clopen and, consequently, the mapping $\psi$ becomes continuous. (The continuity of $\psi$ is obviously equivalent to that of the mapping $x \rightarrow t_{x}$ and the continuity of the latter mapping was pointed out by Dellacherie and Meyer [8, p. 375].) It is easy to check for this altered version of Example 8.5 that $\Gamma_{2}$ is a continuous mapping from $F$ to the space of compact subsets of $\mathscr{P}(F)$ equipped with the Vietoris topology and that $u_{2}$ is also continuous. The same argument as before shows that the measurability of $U^{2}$ is equivalent to that of the PCA set $P$. Thus, even for "continuous problems," the measurability of $U$ is undecidable in general.

In Example 8.5, the function $U^{2}$ is the least excessive majorant of $u$ in the sense of Dubins and Savage [11, Corollary 2.14.1] and no gambler can achieve more even if allowed to use nonmeasurable $\pi$. The next example shows that it is consistent that a gambler can do better with nonmeasurable strategies even if the payoff is calculated using countably additive extensions of the original gambles and, in particular, that the optimal return function as originally defined by Dubins and Savage can be strictly greater than the $U$ of this paper.

Example 8.6. This example will be a modification of a particular instance of Example 8.5 in which the set $P$ is not Lebesgue measurable. To obtain such a set we assume Gödel's axiom of constructibility (cf. Moschovakis [19, p. 279]). It follows that there is a PCA set $P \subseteq I$ such that

$$
\lambda_{*}(P)=\alpha<\beta=\lambda^{*}(P)
$$

where $\lambda$ is Lebesgue measure on $[0,1]$. Let $\left(F_{2}, \Gamma_{2}, u_{2}\right)$ be an instance of Example 8.5 in which this $P$ satisfies (8.2). Modify the example by taking $F_{3}=F_{2} \cup\{p\}$ where $p$ is a point outside $F_{2}$. Define $u_{3}$ and $\Gamma_{3}$ to be the restrictions of $u_{2}$ and $\Gamma_{2}$ on $F_{2}$ and set

$$
u_{3}(p)=-1, \quad \Gamma_{3}(p)=\{\delta(p), \lambda\} .
$$


Then $\left(F_{3}, \Gamma_{3}, u_{3}\right)$ is a Borel problem and, as will now be shown,

$$
U^{3}(p) \leq-1+\alpha .
$$

First notice that, for $x \in I$

$$
\begin{aligned}
U^{3}(x)=U^{2}(x) & =0 & & \text { if } x \in P, \\
& =-1 & & \text { if } x \notin P,
\end{aligned}
$$

and, for any Borel function $f: I \rightarrow R$ such that $f \leq U^{2}$ on $I$,

$$
\int f d \lambda \leq-1+\alpha
$$

by (8.3). Thus, for $\pi=(\sigma, t)$ a Borel policy at $p$ with $\sigma_{0}=\lambda$,

$$
u(\pi)=\int u(\sigma[x], t[x]) \lambda(d x) \leq-1+\alpha,
$$

which establishes (8.4).

Next we will find a nonmeasurable $\pi^{*}$ at $p$ with $u\left(\pi^{*}\right)>U^{3}(p)$.

Let $\varepsilon<\beta-\alpha$.

Choose an extension $\gamma$ of $\lambda$ such that $\gamma(P)=\beta$. Then, for each $x \in I$, choose $\pi_{x}=\left(\sigma_{x}, t_{x}\right)$ at $x$ such that

$$
u\left(\pi_{x}\right) \geq U^{3}(x)-\varepsilon .
$$

(This cannot be a measurable selection.) Define $\sigma_{0}^{*}=\gamma$ and $\sigma^{*}[x]=\sigma_{x}$, and $t^{*}[x]=t_{x}$ for each $x \in I$. Then

$$
u\left(\pi^{*}\right)=\int u\left(\pi_{x}\right) \gamma(d x) \geq-1+\beta-\varepsilon>-1+\alpha \geq U^{3}(p) .
$$

The next example illustrates the possibility that $U$ may be measurable but not excessive. Thus, even if $U$ is measurable, it can nevertheless be smaller than the return function of Dubins and Savage. The example also illustrates that the reduced function may exist and be different from $U$.

Example 8.7. This example will be a modification of another instance of Example 8.5. Assume once more Gödel's axiom of constructibility so that there will exist a function $g: I \rightarrow I$ whose graph $G$ is a coanalytic subset of $X=I \times I$ and contains no uncountable Borel subset of $X[19$, p. 279]. Take the analytic set $A$ of Example 8.5 to be $X \backslash G$ so that $P=I$. Notice that $U^{2}$ is measurable; indeed, $U^{2}$ is identically zero on $I$.

Now define the modified problem $\left(F_{3}, \Gamma_{3}, u_{3}\right)$ exactly as in Example 8.6. Then $U^{3}(p)=-1$ because, for any Borel policy $\pi=(\sigma, t)$ at $p$ with $\sigma_{0} \neq$ $\delta(p)$,

$$
u(\pi) \leq-1+\lambda(E)
$$

where $E=\left\{x \in I: \sigma_{1}(x)(G)=1\right\}$. 
Now $E$ is coanalytic and, consequently, $\lambda(E)=0$. For if $\lambda(E)$ were strictly positive it would contain an uncountable Borel set $B$ and the set

$$
\widetilde{B}=\left\{(x, y): x \in B, \sigma_{1}(x)=\delta(x, y)\right\}
$$

would be an uncountable Borel subset of $G$. It follows that the optimal Borel policy at $p$ is to stagnate immediately.

Finally, observe that $\lambda U^{3}=\lambda U^{2}=0>-1$ so that $U^{3}$ is not excessive, though universally measurable.

Recall that, for a Borel (or analytic) gambling problem, the sets $[U>a]$ are PCA sets. Our final example, which is primarily of interest to set theorists rather than gamblers, will show that every PCA set is of the form $[U>a]$. Thus Theorem 6.3 and the following example together yield a new characterization of PCA subsets of Polish spaces. Another characterization is in Becker [1].

Example 8.8. This will be another modification of Example 8.5, but this time the fortune space $F_{3}$ is taken to be just $F_{2}$. We will modify $u_{2}$ and $\Gamma_{2}$ so that $[U>0]=P$. If $P$ is empty, no change is necessary. If not, choose a point $p \in P$ and set $u_{3}(p)=1$. Let $u_{3}=u_{2}$ on $F_{3} \backslash\{p\}$. Next define

$$
\Gamma_{3}\left(x_{1}\right)=\left\{\delta\left(x_{1}\right)\right\} \cup\left\{\frac{1}{2}\left(\delta(p)+\delta\left(x_{1}, x_{2}\right)\right): x_{2} \in I\right\}
$$

for $x_{1} \in I$, and let $\Gamma_{3}$ restricted to $F_{1}=F_{2} \backslash I$ be $\Gamma_{1}$ as before. The effect of this change is that a gambler beginning at $x \in I$ can now receive an additional half unit of utility (a whole unit if $x=p$ ) and, hence, $\left[U^{3}>0\right]=I \cap\left[U^{2} \geq\right.$ $0]=P$.

\section{AN ALTERNATIVE APPROACH USING}

\section{ALMOST SURELY FINITE STOPPING TIMES}

In the formulation of $\S 1$, the gambler starting at $x$ is required to choose $\sigma \in \Sigma(x)$ and a stop rule $t$ which stops on every history. Now we will allow the gambler to choose a Borel $t: H \rightarrow\{1,2, \ldots\} \cup\{\infty\}$ and such that $[t \leq n]$ is in the sigma-field generated by the first $n$ coordinate functions for $n=1,2, \ldots$ and $\sigma[t<\infty]=1$. Let $T(\sigma)$ be the collection of all such $t$ and define a new optimal return function $W$ by

$$
W(x)=\sup \left\{\int u\left(h_{t}\right) d \sigma: \sigma \in \Sigma(x), t \in T(\sigma)\right\} .
$$

It is assumed in this section that all the integrals occurring in the definition of $W$ exist and that $W$ is everywhere finite. Here is a simple example which shows that $W$ may be strictly greater than $U$ and may not be the reduced function of $u$.

Example 9.1. Let $F=\{0,1, \ldots\} ; u(x)=-x$ for all $x ; \Gamma(0)=\{\delta(0)\}$, $\Gamma(x)=\left\{\delta(x), \frac{1}{2} \delta(x-1)+\frac{1}{2} \delta(x+1)\right\}$ for $x \neq 0$. Then $W(x)=0$ for all $x$ since the simple random walk starting at $x$ will reach 0 almost surely. However, $U=u$ because $u(\pi)=u(x)$ for every $\pi$ at $x$, as follows from 
the optional sampling theorem for everywhere finite stop rules (cf. Dubins and Savage [11, Theorem 2.12.2] or Ramakrishnan and Sudderth [21, Remark 2]).

The next theorem gives a sufficient condition for the equality of the two return functions.

Theorem 9.2. For a given $x \in F$ and every $\varepsilon>0$, suppose there exist $\sigma \in \Sigma(x)$, $t \in T(\sigma)$ such that $\int u\left(h_{t}\right) d \sigma>W(x)-\varepsilon$ and $\left\{u^{-}\left(h_{n}\right)\right\}$ is uniformly integrable under $\sigma$. Then $W(x)=U(x)$.

Proof. The same argument as for Lemma 4.6 shows

$$
\int u\left(h_{t}\right) d \sigma \leq \sup u(\sigma, t \wedge n) \leq U(x)
$$

whenever $\left\{u^{-}\left(h_{n}\right)\right\}$ is uniformly integrable under $\sigma$.

Unlike $U$, the function $W$ is always universally measurable.

Theorem 9.3. $W$ is upper analytic.

The proof requires the study of the set $\mathscr{P}_{\text {as }}$ of almost surely stagnant probability measures on $H$. The definition of $\mathscr{P}_{\text {as }}$ is similar to that of the collections $\mathscr{P}_{\alpha}$ of $\S 4$. For $\sigma \in \mathscr{P}(H), t \in T(\sigma)$, let $\sigma^{t}=\sigma \varphi_{t}^{-1}$ where $\varphi_{t}$ is defined by (4.4). Now set

$$
\mathscr{P}_{\text {as }}=\left\{\sigma^{t}: \sigma \in \mathscr{P}(H), t \in T(\sigma)\right\} .
$$

An alternative description of $\mathscr{P}_{\text {as }}$ is useful. To formulate it, define $\sigma\left[x_{1}, \ldots, x_{n}\right]$ to be a version of the conditional $\sigma$-distribution of $\left(h_{n+1}, \ldots\right)$ given $h_{1}=$ $x_{1}, \ldots, h_{n}=x_{n}$.

Lemma 9.4. A measure $\mu$ belongs to $\mathscr{P}_{\text {as }}$ if and only if

$$
\mu\left\{h: \mu\left[h_{1}, \ldots, h_{n}\right]=\Delta\left(h_{n}\right)\right\} \rightarrow 1 \text { as } n \rightarrow \infty .
$$

Proof. The condition is clearly satisfied by any $\mu \in \mathscr{P}_{\text {as }}$. So assume the condition holds and assume also that, as in Lemma $2.2, \mu\left[h_{1}, \ldots, h_{n}\right]$ is a Borel version. Take

$$
t(h)=\inf \left\{n: \mu\left[h_{1}, \ldots, h_{n}\right]=\Delta\left(h_{n}\right)\right\} .
$$

Then $t \in T(\mu)$ and $\mu=\mu^{t}$. So $\mu \in \mathscr{P}_{\text {as }}$.

Lemma 9.5. $\mathscr{P}_{\text {as }}$ is Borel.

Proof. Use Lemma 2.1 and Lemma 9.4.

Proof of Theorem 9.3. Recall that

Thus, for $x \in F$,

$$
u^{*}(h)=\lim _{n} \sup u\left(h_{n}\right) .
$$

$$
\begin{aligned}
W(x) & =\sup \left\{\sigma^{t} u^{*}: \sigma \in \Sigma(x), t \in T(\sigma)\right\} \\
& =\sup \left\{\sigma u^{*}: \sigma \in \Sigma(x) \cap \mathscr{P}_{\text {as }}\right\} .
\end{aligned}
$$

Now argue as in the proof of Theorem 4.3. 
Example 9.1 shows that $W$ need not be the reduced function of $u$. However, $W$ does have a similar property, as will now be explained.

Say that a universally measurable function $Q: F \rightarrow R$ is strongly excessive if, for every $x \in F, \sigma \in \Sigma(x)$, and $t \in T(\sigma)$,

$$
\int Q\left(h_{t}\right) d \sigma \leq Q(x) \text {. }
$$

Our final theorem is analogous to Theorem 1 of Dubins and Sudderth [12] and the proof is similar also.

Theorem 9.6. The function $W$ is the least universally measurable function $Q: F$ $\rightarrow R$ such that (i) $Q \geq u$ and (ii) $Q$ is strongly excessive.

Proof. Suppose $Q$ is a function having the properties listed and let $x \in F$, $\sigma \in \Sigma(x)$, and $t \in T(\sigma)$. Then

$$
\int u\left(h_{t}\right) d \sigma \leq \int Q\left(h_{t}\right) d \sigma \leq Q(x) .
$$

Take the supremum over $(\sigma, t)$ to see that $W(x) \leq Q(x)$.

It remains to be shown that $W$ has the properties. By Theorem 9.3, $W$ is universally measurable and $W \geq u$ because $\Gamma$ is leavable. We need a lemma to see that $W$ is strongly excessive.

Lemma 9.7. Let $\varepsilon>0$. There exists a universally measurable mapping $\bar{\sigma}: F \rightarrow$ $\mathscr{P}(H)$ such that $\bar{\sigma}(x) \in \Sigma(x) \cap \mathscr{P}_{\text {as }}$ and $\bar{\sigma}(x) u^{*}>W(x)-\varepsilon$ for all $x$.

Proof. The proof is similar to that of Lemma 4.4 in [12].

Now let $x \in F, \sigma \in \Sigma(x), t \in T(\sigma), \varepsilon>0$, and let $\bar{\sigma}$ be given by the lemma. Define $\hat{\sigma} \in \mathscr{P}(H)$ to agree with $\sigma$ on the pre- $t$ sigma-field $\underset{\sim}{F_{t}}$ and to have conditional distribution $\bar{\sigma}\left(h_{t}\right)$ for $\left(h_{t+1}, h_{t+2}, \ldots\right)$ given $\underset{\sim}{F}$. Then it can be checked that $\hat{\sigma} \in \Sigma(x) \cap \mathscr{P}_{\text {as }}$ and, hence,

$$
\begin{aligned}
\int W\left(h_{t}\right) d \sigma & \leq \int \bar{\sigma}\left(h_{t}\right) u^{*} d \sigma+\varepsilon \\
& =\hat{\sigma} u^{*}+\varepsilon \leq W(x)+\varepsilon .
\end{aligned}
$$

This completes the proof of Theorem 9.6.

\section{REFERENCES}

1. H. Becker, Pointwise limits of subsequences and $\Sigma_{2}^{1}$ sets, Fund. Math. 128 (1987), 159-170.

2. D. Blackwell, D. Freedman, and M. Orkin, The optimal reward operator in dynamic programming, Ann. Probab. 2 (1974), 926-941.

3. D. Blackwell and S. Ramakrishnan, Stationary plans need not be uniformly adequate for leavable, Borel gambling problems, Proc. Amer. Math. Soc. 102 (1988), 1024-1027.

4. C. Dellacherie, Les dérivations en théorie descriptive des ensembles et le théorème de la borne, Séminaire de Probabilités, XI (Strasbourg 1975-76), Lecture Notes in Math., vol. 581, Springer-Verlag, Berlin and New York, 1977, pp. 34-46. (Erratum and addendum, Séminaire de Probabilités XII, Lecture Notes in Math., vol. 649, Springer-Verlag, 1978, p. 523.) 
5. __ Transformations analytiques: théorèmes de capacitabilité, de séparation et d'itération transfinie, Séminaire Initiation à l'Analyse 20, Publ. Math. Univ. Pierre et Marie Curie 46 (1980-81), 16-01-16-27.

6. __ Quelques résultats sur les maisons de jeux analytiques, Séminaire de Probabilités XIX (Strasbourg 1983-84), Lecture Notes in Math., vol. 1123, Springer-Verlag, Berlin and New York, 1985, pp. 222-229.

7. __ Les sous-noyaux élémentaires, Théorie du Potentiel Proceedings (Orsay 1983), Lecture Notes in Math., vol. 1096, Springer-Verlag, Berlin and New York, 1983, pp. 183-222.

8. C. Dellacherie and P. A. Meyer, Ensembles analytiques et temps d'arrêt, Séminaire de Probabilités IX (Strasbourg 1973-74), Lecture Notes in Math., vol. 465, Springer-Verlag, Berlin and New York, 1975, pp. 373-389.

9. __ Probabilités et potentiel, Hermann, Paris, Chapters I-IV, 1975; Chapters IX-XI, 1983.

10. L. E. Dubins and D. A. Freedman, Measurable sets of measures, Pacific J. Math. 14 (1964), 1211-1222.

11. L. E. Dubins and L. J. Savage, Inequalities for stochastic processes, Dover, New York, 1976.

12. L. E. Dubins and W. D. Sudderth, Countably additive gambling and optimal stopping, Z. Wahrsch. Verw. Gebiete 41 (1977), 59-72.

13. $\ldots$, On stationary strategies for absolutely continuous houses, Ann. Probab. 7 (1979), 461-476.

14. K. Kuratowski, Topology, vol. 1, Academic Press, New York, 1966.

15. A. Louveau, Capacitabilité et sélections Boreliennes, Séminaire Initiation à l'Analyse 21, Publ. Math. Univ. Pierre et Marie Curie 54 (1981-82), 19-01-19-21.

16. A. Maitra, V. Pestien, and S. Ramakrishnan, Domination by Borel stopping times and some separation properties, Fund. Math. (to appear).

17. D. A. Martin and R. M. Solovay, Internal Cohen extensions, Ann. Math. Logic 2 (1970), 143-178.

18. P. A. Meyer and M. Traki, Réduites et jeux de hasard, Séminaire de Probabilités VII (Strasbourg 1971-72), Lecture Notes in Math., vol. 321, Springer-Verlag, Berlin and New York, 1973, pp. 155-171.

19. Y. N. Moschovakis, Descriptive set theory, North-Holland, Amsterdam, 1980.

20. K. R. Parthasarathy, Probability measures on metric spaces, Academic Press, New York, 1967.

21. S. Ramakrishnan and W. D. Sudderth, The expected value of an everywhere stopped martingale, Ann. Probab. 14 (1986), 1075-1079.

22. R. E. Strauch, Measurable gambling houses, Trans. Amer. Math. Soc. 126 (1967), 64-72. (Correction, 130 (1968), 184.)

23. W. D. Sudderth, On the existence of good stationary strategies, Trans. Amer. Math. Soc. 135 (1969), 399-414.

School of Statistics, University of Minnesota, Minneapolis, Minnesota 55455 (Current address of A. Maitra and W. Sudderth)

Department of Statistics, University of California, Berkeley, California 94720 (Current address of $R$. Purves) 